\title{
Chronic colitis promotes tumor development
}

\author{
MASARU SHINOZAKI ${ }^{1}$, TOSHIAKI WATANABE ${ }^{1}$, HAJIME SATO $^{2}$ and HIROKAZU NAGAWA ${ }^{1}$ \\ ${ }^{1}$ Departments of Surgical Oncology, and ${ }^{2}$ Public Health and Occupational Medicine, \\ Graduate School of Medicine, The University of Tokyo, Japan
}

Received December 5, 2005; Accepted February 9, 2006

\begin{abstract}
Patients with chronic ulcerative colitis have a significantly increased risk of colorectal cancer development. This study was aimed at clarifying whether colitis promotes tumor development or not. A dose of $200 \mathrm{mg} / \mathrm{kg}$ body weight 1,2-dimethylhydrazine was given to male Wistar rats. Four weeks later, $5 \%$ acetic acid (colitis group) or $0.9 \%$ saline (control group) was administered intrarectally once a week for 12 weeks and the rats were sacrificed after 27 weeks of dimethylhydrazine injection. Macroscopic lesions (ML) were more frequently detected in the colitis group than in the control group without statistical significance. However, the number of ML per rat with ML was largest in the colitis group ( 4.50 vs. $1.33 ; p=0.039$ ). Eleven of 13 tumors were sessile in the colitis group, while three of five were pedunculated in the control group ( $\mathrm{p}=0.044)$. All ML of $3 \mathrm{~mm}$ or more in diameter in the control group were intramucosal well-differentiated tumors. In the colitis group, 4 of 13 tumors were poorly or moderately differentiated or mucinous carcinomas, and 11 of 13 invaded the submucosal layer or deeper $(p=0.003)$. The number of aberrant crypt foci per rat was smaller in the colitis group than in the control group. The number of crypt orifices was larger in the colitis group than in the control group (23.6 vs. 8.8: $\mathrm{p}<0.001)$. Significantly higher proliferative activity of normal-appearing mucosa was noted in the colitis group in all three parts of the colon. Colitis is suggested to promote colonic tumor development.
\end{abstract}

\section{Introduction}

Although a significantly increased risk of colorectal cancer is recognized in patients with chronic ulcerative colitis (UC) in comparison with the background population (1), the role of $\mathrm{UC}$ in the development of colorectal cancer remains unknown (2). Some researchers have hypothesized that a genetic factor (3), folate deficiency (4), or extraintestinal manifestations of

Correspondence to: Dr Masaru Shinozaki, Present address: Department of Surgery, Omori Red Cross Hospital, 4-30-11 Chuo Ota-ku, Tokyo 143-8527, Japan

E-mail: mshino@zb4.so-net.ne.jp

Key words: colitis, cancer, promotion, carcinogenesis
UC, such as cholestatic liver disease (5), are involved in the etiology of this cancer. As chronic inflammation of other organs, e.g. esophagitis (6), cholecystitis (7), and gastritis (8) is associated with an increased incidence of cancer, it has been suggested that UC-associated carcinogenesis is related to the inflammatory disease process itself (9).

Carcinogenesis consists of at least three processes: initiation, promotion and progression (10), and the mechanism by which colitis influences tumor generation has not yet been determined. It is speculated that the effect of inflammation on carcinogenesis may be due to increased initiation; i.e., in the presence of inflammation, increased cell turnover enhances the possibility of genetic transcriptional errors, which increases the chance of tumorigenesis. Another hypothesis is that inflammation promotes tumor development, especially from subclinical lesions to life-threatening tumors.

To determine whether colitis promotes tumor progression or not, we investigated the influence of repeated acetate enema-induced chronic colitis on tumors induced by $1,2-$ dimethylhydrazine (DMH) injection.

\section{Animals and methods}

Animals and study design. Twenty-one male Wistar rats aged 4 weeks were purchased from Charles River Japan (Yokohama, Japan). They were individually kept in aluminum wire cages. A week later, $200 \mathrm{mg} / \mathrm{kg}$ body weight of DMH (Wako Chemical Industries, Osaka, Japan) was administered to the rats, which were randomly allocated to two groups: colitis group and control group. Four weeks later, $6 \mathrm{ml} / \mathrm{kg} 5 \%$ acetic acid (colitis group) or $0.9 \%$ saline (control group) was administered intrarectally via an 8-cm silicone catheter once a week for 12 consecutive weeks. At 27 weeks after DMH injection, rats were intraperitoneally injected with $10 \mathrm{mg} / \mathrm{kg}$ bromodeoxyuridine (Sigma, USA) and, 30 min later, they were sacrificed. The colon, liver, and lymph nodes near the colon were excised. The colon was opened by a longitudinal incision, pinned out on a cork board, and fixed in $10 \%$ formaldehyde.

All animals were treated in accordance with the guide for animal experimentation of the Faculty of Medicine, the University of Tokyo.

Surface examination. The size of macroscopic lesions (ML; visible with the naked eye) was expressed as the diameter in $\mathrm{mm}$. The mucosal surface was stained with $0.1 \%$ methylene blue and examined with a stereomicroscope (Olympus, Tokyo, Japan). Aberrant crypt foci (ACF: Fig. 1) were identified by 


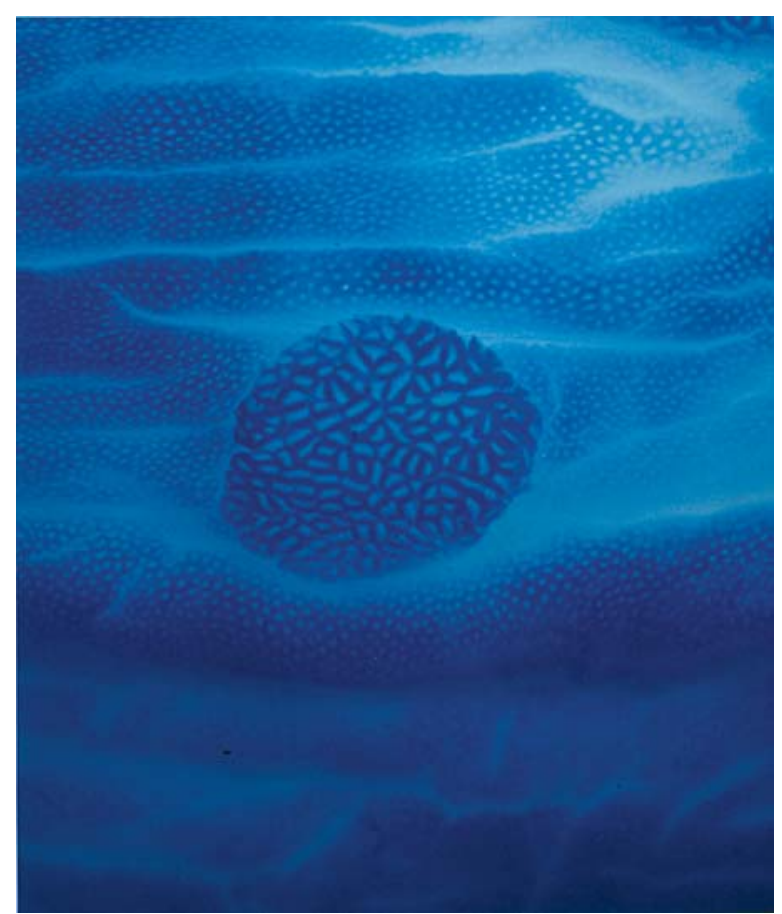

Figure 1. Aberrant crypt foci.

abnormality of the crypt orifice. ACF size was expressed as the number of crypt orifices.

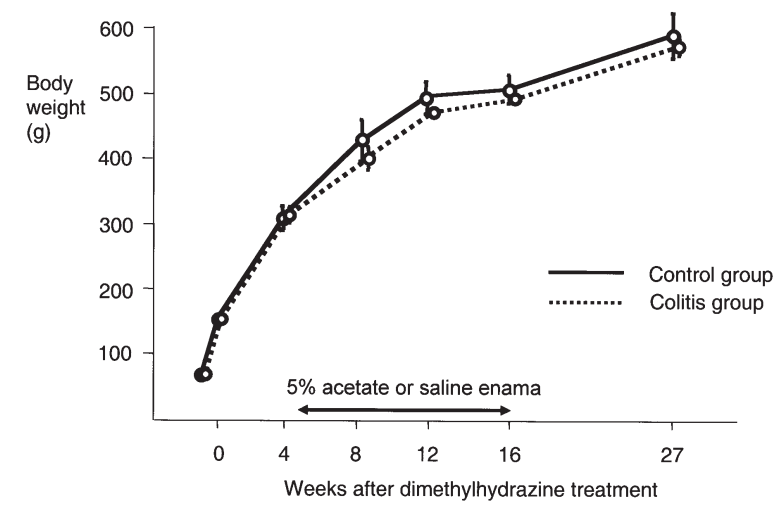

Figure 2. Time course of changes in body weight. Although body weight was nearly the same in the two groups before colitis induction, rats in the control group tended to be heavier than those in the colitis group thereafter, but the difference did not reach statistical significance.

Histological examination. All tumors with a diameter of $3 \mathrm{~mm}$ or more were sectioned and stained with hematoxylin and eosin. The differentiation of tumors and depth of invasion were recorded according to the WHO classification for colorectal cancer.

Bromodeoxyuridine staining. Four- $\mu$ m-thick sections were deparaffinized in xylene and rehydrated in decreasing concentrations of ethanol. After $0.1 \%$ trypsin digestion for $20 \mathrm{~min}$ at $37^{\circ} \mathrm{C}$, the slides were incubated in $95 \%$ formamide

A

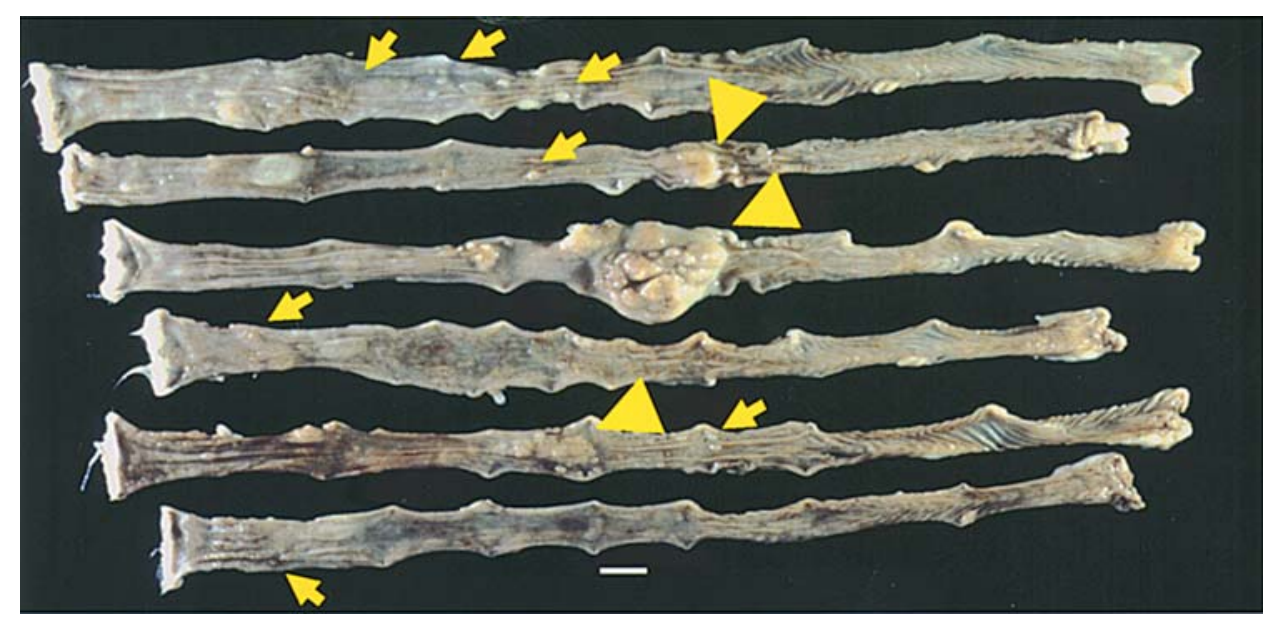

B

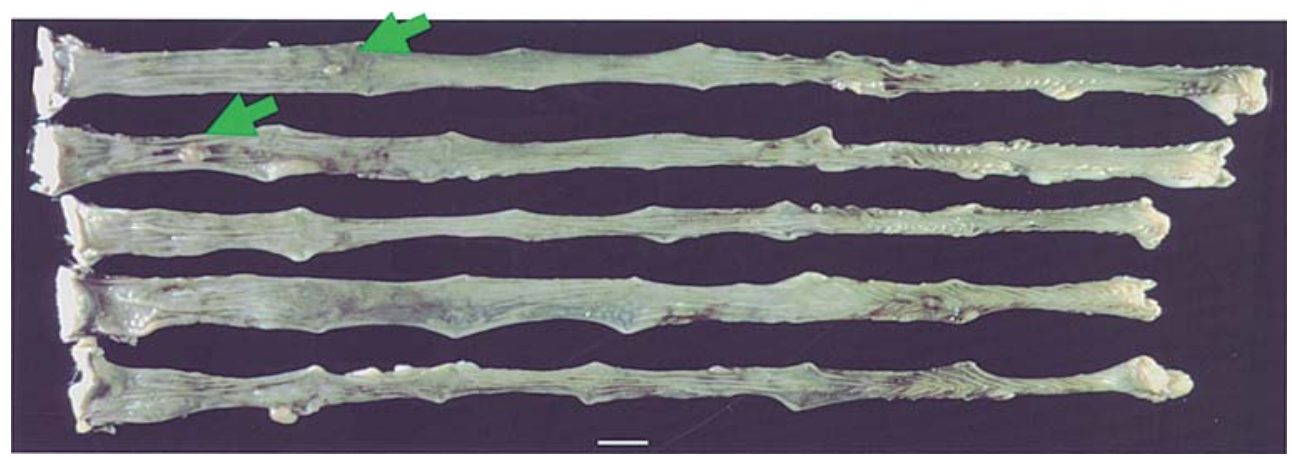

Figure 3. Macroscopic lesions. (A) Colitis group, rats had larger sessile tumors mainly in the middle colon. (B) Control group, rats had smaller pedunculated tumors in the distal colon. 
Table I. Number of macroscopic lesions ( $\geq 3 \mathrm{~mm}$ ) according to pathological characteristics.

Colitis group Control group $\mathrm{p}$ value $\mathrm{a}^{\mathrm{a}}$

\begin{tabular}{lrrr}
\hline $\begin{array}{l}\text { Pedunculation } \\
\text { Absent }\end{array}$ & 12 & 2 & 0.044 \\
Present & 1 & 3 & \\
Submucosal invasion & & & 0.003 \\
Absent & 2 & 5 & \\
Present & 11 & 0 & \\
Histology & & & 0.180 \\
Well differentiated & 9 & 5 & \\
Moderately differentiated & 1 & 0 & \\
Poorly differentiated & 1 & 0 & \\
Mucinous carcinoma & 2 & 0 & \\
Total & 13 & 5 & \\
\hline
\end{tabular}

${ }^{\mathrm{a} F i s h e r}$ 's exact test was used for Pedunculation and Submucosal invasion, and Cuzick test was used for Histology

in $0.15 \mathrm{M}$ trisodium citrate at $70^{\circ} \mathrm{C}$ for $45 \mathrm{~min}$ for partial denaturation of double-stranded DNA. Immunostaining was performed with anti-bromodeoxyuridine antibody (Dako Japan, Tokyo, Japan) at a dilution of 1:20 at $4^{\circ} \mathrm{C}$ using a Histofine immunostaining kit (Nichirei, Tokyo, Japan), according to the manufacturer's instructions.

Proliferative activity. Proliferative activity was expressed as the ratio of positively-stained cells in bromodeoxyuridineimmunostained slides. For normal-appearing epithelium, welloriented crypts were counted. For tumors, the ratio of stained neoplastic cells to all neoplastic cells in randomly selected fields was expressed as proliferative activity. All slides were coded and read by investigators blinded to their identity.

Statistics. Numeric data were expressed as mean \pm standard deviation. Mann-Whitney's U-test was performed for numeric data, and Fisher's exact test (two group comparison) or Cuzick test (more than two group comparison) was performed for categorical data using Statview version 4.5J on a Macintosh computer. A level of $\mathrm{p}<0.05$ was considered statistically significant.

\section{Results}

Survival and body weight (Fig. 2). Only one rat in the control group died, on the day after DMH injection, and was excluded from the study. No other rat died before sacrifice. Although body weight was nearly the same in the two groups before colitis induction, rats in the control group tended to be heavier than those in the colitis group thereafter, but the difference did not reach statistical significance.

Macroscopic lesions and aberrant crypt foci. Representative specimens are shown in Fig. 3. More than $90 \%$ of rats in the

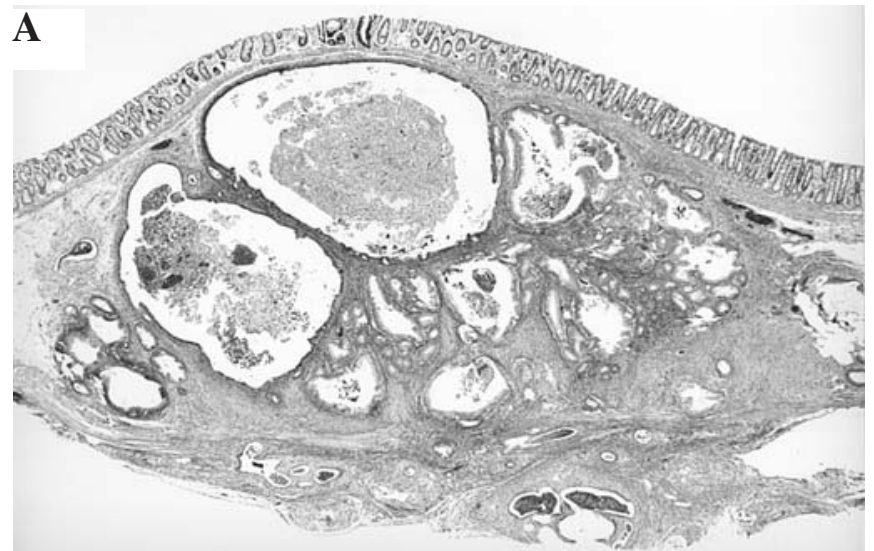

B

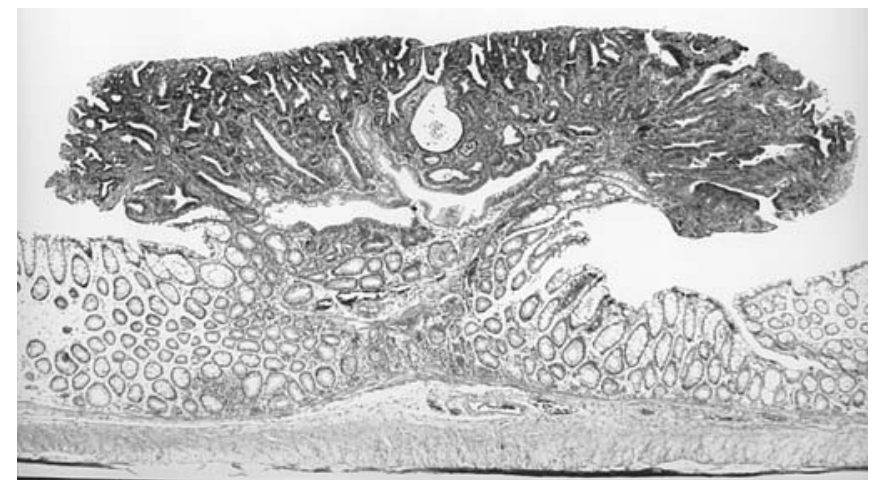

Figure 4. Microscopic study of lesions. (A) Colitis group, mucinous or poorly differentiated carcinoma was detected. Invasive cancer was found only in the colitis group. Most of the lesions were sessile. (B) Control group, only well-differentiated, intramucosal tumors were found. Pedunculated lesions were often seen.

colitis group had ML, whereas two thirds of rats in the control group had ML, but the difference did not reach statistical significance $(\mathrm{p}=0.285)$. The number of ML per rat with $M L$ was significantly larger in the colitis group than in the control group ( $4.50 \pm 5.32$ vs. $1.33 \pm 0.52$, respectively; $\mathrm{p}=0.039)$. Thus, we found more ML in colitis rats than in the control group.

All rats in both groups showed ACF. The number of ACF per rat tended to be smaller in the colitis group than in the control group (14.3 \pm 8.13 vs. $25.6 \pm 11.0 ; \mathrm{p}=0.068)$.

The average size of ML was essentially the same in the two groups. However, there were four large tumors with a diameter of $10 \mathrm{~mm}$ or more, all of which belonged to the colitis group. The proportion of ML in all tumors (the sum of ML and ACF) was significantly higher in the colitis group than in the control group $(\mathrm{p}=0.0023)$. ACF in the colitis group were significantly larger than those in the control group (23.6 \pm 19.9 vs. $8.78 \pm 8.19 ; \mathrm{p}<0.001)$. Therefore, colitis was suggested to make ACF larger, and some of the ACF became visible, which resulted in the presence of larger ML.

Pathological characteristics of tumors (Table I, Fig. 3). The proportion of sessile tumors was significantly higher in the colitis group than in the control group ( $92 \%$ vs. $40 \%$; $=0.044)$. In the control group, all ML were intramucosal, welldifferentiated neoplasms whereas, in the colitis group, 4 of 12 tumors were histologically poorly differentiated, moderately differentiated or mucinous carcinoma. While all ML were 
A

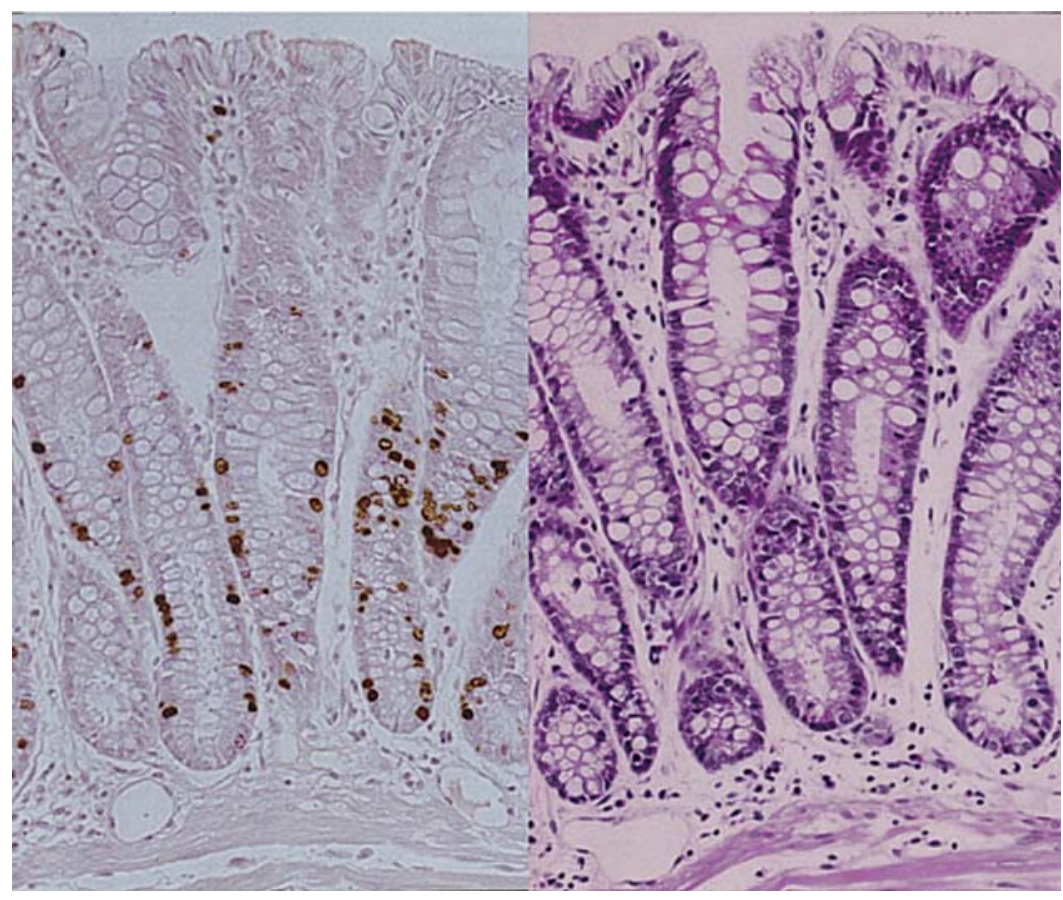

B

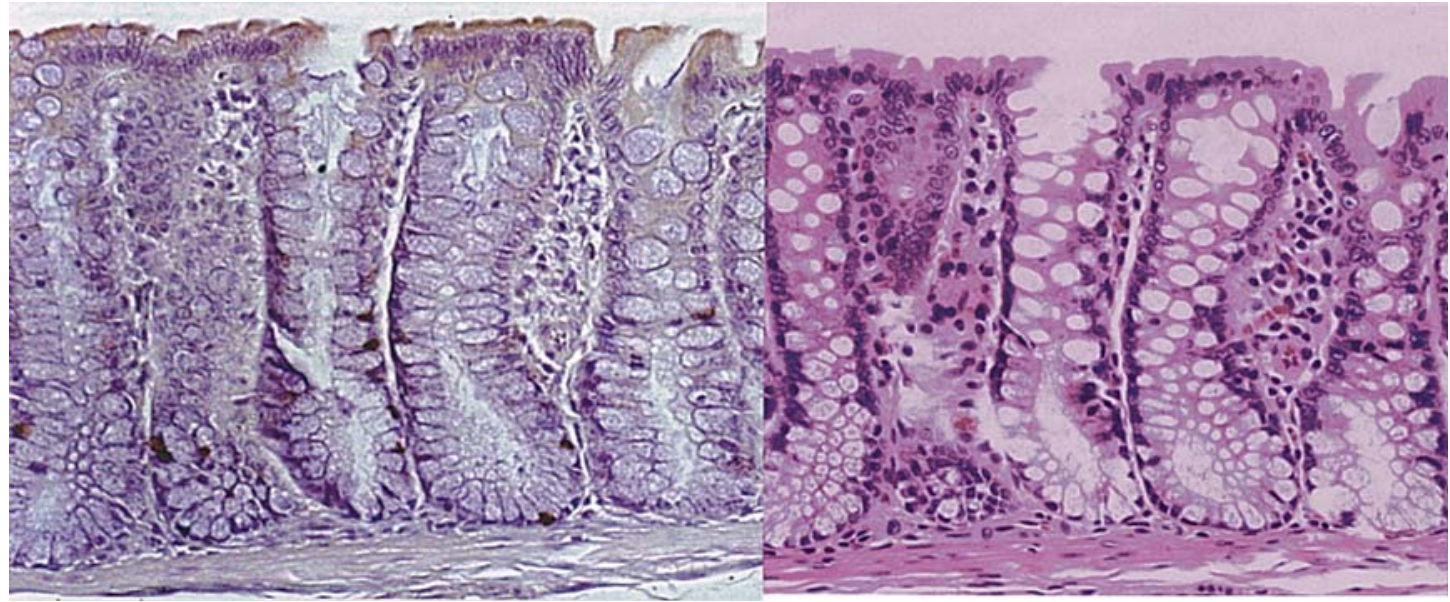

Figure 5. Bromodeoxyuridine staining. A higher proportion of normally appearing epithelial cells was immunostained with anti-bromodeoxyuridine antibody in the colitis group (A) than that in the control group (B). Left, bromodeoxyuridine staining; right, hematoxylin and eosin staining.

intramucosal in the control group, 10 of $12(83 \%)$ tumors were invasive cancer in the colitis group. This difference was statistically significant $(\mathrm{p}=0.003)$.

Proliferative activity (Fig. 4). Normal-appearing mucosa showed significantly higher proliferative activity in colitis rats than in control rats $(0.063 \pm 0.045$ vs. $0.027 \pm 0.027 ; \mathrm{p}<0.0001)$. Although bromodeoxyuridine labeling index of tumors in the colitis group was higher than that in the control group $(0.049 \pm 0.077$ vs. $0.022 \pm 0.025)$, the difference did not reach statistical significance $(\mathrm{p}=0.78)$.

\section{Discussion}

In the present study, the number of ML was increased in the colitis group. On the other hand, the total number of lesions did not differ significantly, or was even smaller in the colitis group. This increase in number of ML can not be explained by increased chance of initiation. Furthermore, large ML ( $>10 \mathrm{~mm}$ in diameter), a high frequency of ML, and larger $\mathrm{ACF}$ were seen in the colitis group. We interpret this increase in size of macroscopic and microscopic lesions without a change in the total number of lesions as promotion of carcinogenesis by colitis. Our results showed that invasive cancers were detected only in the colitis group, which implies that colitis takes part in the progression of carcinogenesis. Thus, colitis is associated with promotion and progression of tumors in this model.

Bromodeoxyuridine immunostaining in the present study showed that normal-appearing mucosa in the colitis group had significantly higher proliferative activity than that in the control group. As promotion is considered to be related to an increase in the cell cycle (10), this result also supports the hypothesis that colitis promotes colonic carcinogenesis.

Tumors in the colitis group included invasive carcinoma and poorly differentiated adenocarcinoma, which were not 
seen in rats without colitis. Similar findings were reported previously. Long-term administration of dextran sulfate sodium produced mucinous carcinomas in hamsters (11). A mucinous carcinoma was found in a rat treated with simultaneous administration of DMH and dextran sulfate sodium (12). Furthermore, the ratio of mucinous or poorly differentiated cancers is higher in human UC than in sporadic cancer patients (13). Thus, mucinous or poorly differentiated cancers may have a growth advantage in colitis, or histological transformation may be induced by colitis. Further study is needed to clarify the mechanism.

Previous studies have revealed that long-term administration of colitis-inducing agents, such as dextran sulfate sodium (14-16) and degraded carrageenan (17-19), induced colonic tumors. In these models, however, the timing of initiation and promotion can not be distinguished. Moreover, it is quite difficult to exclude that these chemicals are mutagenic because even a small mutagenic effect might cause genetic change in the long term. Another representative model was induced by simultaneous administration of a carcinogen and a colitis inducer (20-22). An inherent weakness of this model is that the increased number of tumors may not be due to colitis itself but to increased carcinogen uptake into colonocyte nuclei in association with the increased cell cycle due to colitis. Carcinogens and colitis-inducing agents should not be administered simultaneously, in order to clarify the influence of colitis on tumor development. Davis et al reported that DMH had been metabolized by $24 \mathrm{~h}$ after injection (23). Therefore, we adopted a model with a single injection of a relatively high dose of DMH followed by repeated acetic acid enemas at intervals of 4 weeks. To our knowledge, no similar protocols have been used previously. In the present study, acetic acid was used to induce colitis because it has a simple chemical structure and is physiologically present in the colonic lumen. DMH was selected as a carcinogen because it is one of the most commonly used substances for generating colonic neoplasia.

Poorly differentiated adenocarcinoma and mucinous carcinoma are more common in UC (13). Furthermore, significantly higher proliferative activity of normal-appearing mucosa is reported in UC patients (24-25), especially in tumorbearing colitis patients (Shinozaki M, et al, Gastroenterology 106: abs. 440, 1994). These characteristics are similar to our model. Therefore, a single DMH injection followed by repeated administration of acetic acid at intervals of 4 weeks is a model that resembles clinical colitic cancers. At the same time, this model provides a relatively high frequency of invasive cancers. We think that this model is also useful to investigate the mechanism of invasion by colorectal cancers in vivo.

In our study, the number of ACF in the colitis group was smaller than that in the control group. This decrease may be attributable to the colitis; i.e., colitis may destroy ACF as well as normal epithelium. Another possible explanation is that this decrease could be due to ML. In the colitis group, very large tumors were present, which were absent in the control group. As ACF were not counted in a large tumor, there may be a difference in the area of non-neoplastic colon between the two groups. A third explanation is that promotion of the lesions may have resulted in a decrease in ACF; i.e. some ACF became larger and were identified as ML, with a decrease in number of ACF.

This study was designed to examine the promoting effect of colitis on carcinogenesis. Therefore, the role of colitis in the initiation of chronic carcinogenesis remains unknown. However, the numbers of ML and ACF in the proximal colon were essentially the same in the two groups, though bromodeoxyuridine labeling index in normal-appearing mucosa was significantly higher in the colitis group. Thus, the influence of colitis on initiation may be smaller than its effect on promotion or progression. Further investigation is required to clarify the process of carcinogenesis in colitic cancer.

In conclusion, acetic acid-induced colitis promotes DMHinduced colonic tumor development. This model may be useful for investigation of carcinogenesis in UC and the mechanism of cancer invasion.

\section{References}

1. Ekbom A, Helmick CG, Zack M, Holmberg L, and Adami HO: Survival and causes of death in patients with inflammatory bowel disease: a population-based study. Gastroenterology 103: 954-960, 1992.

2. Mellemkjaer L, Olsen JH, Frisch M, et al: Cancer in patients with ulcerative colitis. Int J Cancer 60: 330-333, 1995.

3. Gyde SN, Prior P, Allen RN, et al: Colorectal cancer in ulcerative colitis: A cohort study of primary referrals from three centres. Gut 29: 206-217, 1988

4. Lashner BA, Heidenreich PA, Su GL, Kane SV and Hanauer SB: Effect of folate supplementation on the incidence of dysplasia and cancer in chronic ulcerative colitis. Gastroenterology 97 : 255-259, 1989.

5. D'Haens GR, Lashner GR, and Hanauer SB: Pericholangitis and sclerosing cholangitis are risk factors for dysplasia and cancer in ulcerative colitis. Am J Gastroenterol 88: 1174-1178, 1993.

6. Kahrilas PJ: Gastroesophageal reflux disease. JAMA 276: 983-988, 1996.

7. Bansal P and Sonnenberg A: Comorbid occurrence of cholelithiasis and gastrointestinal cancer. Eur J Gastroenterol Hepatol 8: 985-988, 1996.

8. Watanabe Y, Kurata JH, Mizuno S, et al: Helicobacter pylori infection and gastric cancer. A nested case-control study in a rural area of Japan. Dig Dis Sci 42: 1383-1387, 1997.

9. Levin B: Ulcerative colitis and colon cancer: biology and surveillance. J Cell Biochem 16G: 47-50 (suppl), 1992.

10. Pilot HC: Multistage carcinogenesis- Genetic and epigenetic mechanisms in relation to cancer prevention. Cancer Detect Prev 17: 567-563, 1993.

11. Yamada M, Ohkusa T and Okayasu I: Occurrence of dysplasia and adenocarcinoma after experimental chronic ulcerative colitis in hamsters induced by dextran sulfate sodium. Gut 33: 1521-1527, 1992.

12. Hirono I, Ueno I, Aiso S, Yamaji T and Goldberg L: Enhancing effect of dextran sulfate sodium on colorectal carcinogenesis by 1,2-dimethylhydrazine in rats. Gann 74: 493-496, 1983.

13. Connell WR, Talbot IC, Harpaz N, Britto N, Wilkinson KH, Kamm MA and Lennard-Jones JE: Clinicopathological characteristics of colorectal carcinoma complicating ulcerative colitis. Gut 35: 1419-1423, 1994

14. Hirono I, Kuhara K, Hosaka S, Tomizawa S and Goldberg L: Induction of intestinal tumors in rats by dextran sulfate sodium. J Natl Cancer Inst 66: 579-581, 1981.

15. Tamaru T, Kobayashi H, Kishimoto S, Kajiyama G, Shimamoto F and Brown WR: Histochemical study of colonic cancer in experimental colitis of rats. Dig Dis Sci 38: 529-537, 1993.

16. Hirono I, Kuhara K, Yamaji T, Hosaka S and Goldberg L: Induction of colorectal squamous cell carcinoma in rats by dextran sulfate sodium. Carcinogenesis 3: 353-355, 1982.

17. Fabian RJ, Abraham R, Coulston F and Goldberg L: Carrageenaninduced squamous metaplasia of the rectal mucosa in the rat. Gastroenterology 65: 265-276, 1973.

18. Wakabayashi K, Inagaki T, Fujimoto Y and Fukuda Y: Induction by degraded carrageenan of colorectal tumors in rats. Cancer Lett 4: 171-176, 1978. 
19. Kitano A, Matsumoto T, Hiki M, et al: Epithelial dysplasia of the rabbit colon induced by degraded carrageenan. Cancer Res 46: 1374-1376, 1996.

20. Davey PG, MaGuiness EE, Nicol S and Wormsley KG: Experimental colitis and colonic cancer in the rat. Mount Sinai J Med 52: 209-211, 1985.

21. Chester JF, Gaissert HA, Ross JS, Malt RA and Weitzmann SA: Colonic cancer induced by 1,2-dimethylhydrazine: promotion by experimental colitis. Br J Cancer 59: 704-705, 1989.

22. Hagihara PF: Experimental colitis as a promoter in large-bowel tumorigenesis. Arch Surg 117: 1304-1307, 1982.

23. Davis AE, Patterson F and Crouch R: The effect of therapeutic drugs used in inflammatory bowel disease on the incidence and growth of colonic cancer in the dimethylhydrazine rat model. $\mathrm{Br}$ J Cancer 66: 777-780, 1992.
24. Noffsinger AE, Miller MA, Cusi MV and Fenoglio PC: The pattern of cell proliferation in neoplastic and nonneoplastic lesions of ulcerative colitis. Cancer 78: 2307-2312, 1996.

25. Kullmann F, Fadaie M, Gross V, et al: Expression of proliferating cell nuclear antigen (PCNA) and Ki-67 in dysplasia in inflammatory bowel disease. Eur J Gastroenterol Hepatol 8: 371-379, 1996.

26. Shinozaki M, Watanabe T, Kubota Y, Sawada T, Nagawa H and Muto T: High proliferative activity is associated with dysplasia in ulcerative colitis. Dis Colon Rectum 42 (suppl): S34-S39, 2000 . 